Didáctica. Lengua y literatura

ISSN: 1130-0531

http://dx.doi.org/10.5209/DIDA.61954

\title{
Distancia entre lenguas / culturas y transferencia lingüística / cultural: sus efectos en el proceso de adquisición del inglés como lengua extranjera
}

\author{
Richard Clouet ${ }^{1}$
}

Recibido: 12 de enero de 2015 / Aceptado: 15 de febrero de 2015

Resumen. El aprendizaje de una lengua extranjera aparece mediatizado por una serie de factores entre los cuales destacamos la distancia existente entre la lengua y cultura origen y la lengua y cultura meta. En este artículo nuestra pretensión consiste en poner en evidencia que el éxito de la adquisición dependerá de las características del comportamiento lingüístico y cultural del aprendiz y de sus preconocimientos lingüísticos y culturales en los procesos asociativos. El presente estudio empírico se centra en la influencia interlingüística e intercultural de la lengua española en la adquisición del inglés como lengua extranjera. El objetivo central es determinar si la lengua y la cultura materna influyen favorablemente o negativamente en la adquisición de la lengua extranjera. Los sujetos de este estudio son estudiantes hispanófonos del Grado en Traducción e Interpretación de la Universidad de Las Palmas de Gran Canaria. Los datos analizados confirman que mientras la percepción de similitudes entre lenguas y culturas puede facilitar el proceso de enseñanza / aprendizaje, la transferencia lingüística y cultural también puede ser el origen de errores incomprensibles por un hablante nativo.

Palabras clave: Transferencia lingüística; transferencia cultural; distancia entre lenguas; distancia entre culturas; interlengua.

\section{[en] Language / Culture Distance and Linguistic / Cultural Transfer: Its Effects in the Learning Process of English as a Foreign Language}

\begin{abstract}
The learning of a foreign language is influenced by a number of factors including the effects of the distance between the source language and culture, and the target language and culture. In this article our aim is to highlight the fact that successful acquisition will depend on the characteristics of the linguistic and cultural behaviour of the learners, as well as on their associative knowledge related to their linguistic and cultural background. This empirical study focuses on the cross-linguistic and cross-cultural influence of Spanish on the acquisition of English as a foreign language. The central objective is to observe if the mother tongue and culture promote or hinder the acquisition of English as a second language. The subjects in this study are Spanish-speaking students in the first year of the Translation and Interpreting Degree at the University of Las Palmas de Gran Canaria, Spain. The data analysed confirms that while the perception of similarities between languages and cultures can facilitate learning, language and culture transfer may cause non-native forms (errors) to occur.
\end{abstract}

Keywords: Linguistic transfer; cultural transfer; linguistic distance; cultural distance; interlanguage.

1 Departamento de Filología Moderna. Universidad de Las Palmas de Gran Canaria (España)

E-mail richard.clouet@ulpgc.es 
[fr] Distance entre langues / cultures et transferts linguistiques / culturels: leurs effets sur le processus d'acquisition de l'anglais comme langue étrangère

\begin{abstract}
L'apprentissage d'une langue étrangère est influencé par un certain nombre de facteurs parmi lesquels celui de la distance entre la langue / culture source et la langue / culture cible. Dans cet article, notre objectif est de souligner le fait qu'une acquisition réussie dépendra des caractéristiques de comportement linguistique et culturel de l'apprenant, ainsi que des connaissances associatives qu'il possède, celles-ci étant directement liées à leurs origines linguistiques et culturelles. Cette étude empirique porte sur les influences interlinguistiques et interculturelles de la langue espagnole dans l'acquisition de l'anglais comme langue étrangère. L'objectif principal est de déterminer si la langue et la culture maternelles ont une influence positive ou négative sur l'acquisition d'une langue étrangère. Les sujets de cette étude sont des étudiants hispanophones de la Licence en traduction et interprétation à l'Université de Las Palmas de Gran Canaria. Les données analysées confirment que, bien que la perception des similitudes entre les langues et les cultures puisse faciliter le processus d'enseignement / apprentissage, les transferts linguistiques et culturels peuvent aussi être la source d'erreurs incompréhensibles pour un locuteur natif.
\end{abstract}

Keywords: Transfert linguistique ; transfert culturel ; distance entre les langues ; distance entre les cultures ; interlangue.

Sumario. 1. Introducción. 2. Distancia entre lenguas y distancia entre culturas. 3. Transferencia lingüística y transferencia cultural. 4. Estudio empírico. 5. Aplicación didáctica. 6. Discusión y conclusiones. 7. Bibliografía.

Cómo citar: Clouet, L. (2018) Distancia entre lenguas / culturas y transferencia lingüística / cultural: sus efectos en el proceso de adquisición del inglés como lengua extranjera, Didáctica. Lengua y literatura, 30, 57-72.

\title{
1. Introducción
}

La dificultad de aprender y adquirir una lengua extranjera radica sobre todo en la distancia entre las lenguas, las culturas y las visiones del mundo. Las palabras de una lengua se asocian a un sistema de tópicos y de referencias vinculado con su contexto cultural y organizado en un sistema conceptual que refleja una determinada cosmovisión (Soria Clivillés, 1993). Por lo tanto, el estudiante ha de ser sensible a la diferencia cultural y conceptual que se esconde detrás de la lengua meta, y la adquisición de una segunda lengua (en adelante L2) no será óptima ni completa si no se incorporan estos aspectos en la enseñanza de lenguas extranjeras.

Con el término distancia lingüística nos referimos a la diferencia tipológica entre la primera lengua del alumno y la lengua meta, es decir, al grado de semejanza o divergencia que entre ellas se da. También consideraremos la distancia lingüística como una de las condiciones relacionadas con la transferencia. La proximidad o distancia entre lenguas puede influir sobre el aprendizaje tanto a través de la transferencia positiva como de la negativa (interferencia). 


\section{Distancia entre lenguas y distancia entre culturas}

Las investigaciones en la adquisición de segundas lenguas han demostrado, a lo largo de las últimas dos décadas, los efectos de factores como la distancia entre lenguas que, a su vez, también tiene un papel importante en la influencia interlingüística y la comunicación intercultural, puesto que hoy en día parece impensable estudiar la distancia lingüística sin hacer hincapié, previamente, en la distancia cultural.

Dominar una lengua no sólo significa conocer y saber utilizar sus estructuras gramaticales sino también tener conocimientos de la cultura en que está inmersa. Cuanto más se acerque al contexto cultural de pensamiento y de conducta de la lengua extranjera, más fácil será para el alumno utilizarla correctamente y comunicarse con los demás.

La noción de lengua y cultura como binomio indisociable ha sido destacada por numerosos teóricos, entre los que cabe resaltar al romántico alemán Wilhem von Humboldt para quien el lenguaje refleja el espíritu del pueblo. Esta noción de relatividad lingüística fue desarrollada por la llamada hipótesis Sapir / Whorf (Sapir, 1954; Whorf 1956) que establece que el conocimiento que un pueblo tiene del mundo está en relación con su lenguaje. La lengua, pues, es el vehículo mediante el cual nos expresamos y por el cual reivindicamos nuestra pertenencia a una determinada cultura que nos hace ver y concebir el mundo de una manera particular. Por lo tanto, el concepto clave en el estudio de la distancia entre lenguas es el de la distancia entre culturas, o sea, el de la competencia cultural, la cual se ve necesariamente reflejada en el uso que el alumno hace de la lengua extranjera.

La competencia cultural, es decir, los conocimientos (activos y pasivos) necesarios para poder comportarse adecuadamente en las diferentes situaciones (comunicativas) de una comunidad social, se adquiere a través de la socialización, definida por Berger y Luckmann $(1989,130)$ como: "[...] the comprehensive and consistent induction of an individual into the objective world of a society or a sector in it". Cabe subrayar que "objective" aquí se tiene que leer como "dado" para la comunidad cultural correspondiente, desde su perspectiva, en algún momento concreto.

Por regla general, se suele distinguir entre las primeras experiencias sociales del individuo y la posterior introducción a nuevos ámbitos de su sociedad. Las teorías de la socialización parten de que la socialización primaria es decisiva para el individuo, en el sentido de que le "marca" de una forma muy particular, de modo que las socializaciones posteriores (secundarias) no llegan a ejercer sobre él la misma fuerza que la primera (Berger y Luckmann, 1989, 142). De esta manera, el individuo queda "vinculado" de forma específica a su cultura primaria y no puede "deshacerse" de ella por completo a lo largo de los procesos de socialización secundaria.

En la socialización y a través de ella, el individuo aprende las "reglas" de su comunidad cultural, sus normas y convenciones sociales. Esto hace que el comportamiento de los demás sea, hasta cierto punto, calculable y predecible. Sin embargo, la teoría de la socialización destaca que el concepto de "socialización" no contradice la individualidad única de cada persona. La socialización nunca puede conducir a una homogeneización completa de los miembros de una comunidad social y cultural. Como lo recuerdan Berger y Luckmann $(1989,134)$, "No individual internalizes the totality of what is objectivated as reality in his society, not even if the society and its world are relatively simple ones. On the other hand, there are always elements of subjective reality that have not originated in socialization". 
El individuo no desempeña un papel meramente pasivo en relación con su cultura, sino que participa de forma activa. Lo mismo ocurre cuando conoce otras culturas. El proceso de llegar a conocer otras culturas y, en su caso, adaptarse a ellas se ve sujeto a la vinculación a la cultura primaria: las otras culturas se perciben sobre la base de la propia cultura. También cuando llegamos a conocer otra cultura a través de una tercera, sigue siendo válida tal afirmación: en última instancia, la cultura propia constituye la base a partir de la cual percibimos las culturas ajenas.

Extendiendo estas reflexiones al ámbito de la adquisición de segundas lenguas, llegamos a la conclusión de que, aunque el aprendiz haya desarrollado una competencia lingüística y cultural muy amplia en la(s) lengua(s) y cultura(s) ajena(s) con la(s) que trabaja, no llegará a liberarse del todo de la inevitable vinculación a su lengua y cultura primaria.

Llegando a este punto del artículo y antes de proseguir, cabe clarificar el uso que haremos de los términos "primera lengua", "segunda lengua", "lengua materna" y "lengua extranjera". En el presente estudio, de forma general, utilizaremos los términos "primera lengua", o L1, y "lengua materna" sin distinción, y "segunda lengua", o L2, y "lengua extranjera" como forma neutral cubriendo ambos términos, a pesar de que somos conscientes de la diferencia que hace Ellis (1994) entre adquisición de una segunda lengua y adquisición de una lengua extranjera:

In the case of second language acquisition, the language plays an institutional and social role in the community (i.e. it functions as a recognized means of communication among members who speak some other language as their mother tongue). [...] In contrast, foreign language learning takes place in settings where the language plays no major role in the community and is primarily learnt only in the classroom. (Ellis, 1994, 11).

Por lo tanto, cuando hablamos de distancia lingüística y cultural en el proceso de adquisición de segundas lenguas, nos referimos a la diferencia tipológica entre dos o más lenguas, es decir, al grado de semejanza o divergencia cultural, estructural, léxica, etc. que entre ellas se da. En la enseñanza de lenguas, la distancia lingüística alude a la que existe entre la lengua primera del alumno y la lengua meta. La distancia puede ser considerada como un fenómeno meramente lingüístico, según el grado de diferencias lingüísticas objetivas entre dos lenguas; o como un fenómeno psicolingüístico y sociocultural que viene determinado por el modo en el que los aprendices perciben la relación entre su lengua/cultura primera y la lengua / cultura meta.

Algunas corrientes teóricas sobre adquisición de lenguas consideran estas distancias, y especialmente la distancia lingüística, como una de las condiciones relacionadas con la transferencia.

\section{Transferencia lingüística y transferencia cultural}

La proximidad o distancia entre lenguas puede influir sobre el aprendizaje tanto a través de la transferencia positiva como de la negativa (interferencia). En el proceso de adquisición de la L2, el aprendiz hace un vaivén consciente e inconsciente entre la lengua materna y la lengua extranjera, mediando de forma imparable entre las 
lenguas y las culturas, y adoptando características de comportamientos lingüísticos y culturales que, a veces, se aproximan a las características reales de la lengua extranjera y, otras veces, se alejan.

En el proceso de adquisición de una L2 intervienen factores de dos tipos: los externos y los internos. Entre los externos podemos destacar los que dependen del contexto donde tiene lugar el aprendizaje o el contexto social que lo rodea, mientras que los factores internos están directamente relacionados con los procesos mentales que el aprendiz usa para manipular el input lingüístico y transformarlo en conocimiento y output, entre los cuales se encuentran la lengua materna, la motivación, las estrategias de aprendizaje, las estrategias comunicativas, etc.

Numerosos estudios publicados en los años 70 y 80 analizaron los errores que cometían los aprendices de una L2 debido a estos factores externos e internos, y llevaron a Corder a publicar el indispensable libro para todos aquellos que se iban a dedicar al análisis de errores: Error Analysis and Interlanguage (1981). El término "interlengua" había sido acuñado por Selinker una década antes (1972). Corder evidenció que los hábitos lingüísticos de la lengua materna tienden a provocar errores en la L2, lo cual Brown (1994) denominó "interlingual transfer" or "interference". Sin embargo, también puso de manifiesto que algunos de estos errores no venían de la interferencia de la lengua materna, sino de generalizaciones erróneas que los aprendices hacían dentro de la L2, en palabras de Brown (1994) "intralingual transfer".

Varias teorías se desarrollaron entorno al análisis de errores y la transferencia lingüística en el proceso de adquisición de segundas lenguas, como las de Krashen (1985) y su "modelo del monitor" o las de Hatch (1978) y Long (1983) sobre la necesidad de un input comprensible y de la interacción para la adquisición del lenguaje. Sin embargo, en el marco del presente estudio, nos apoyamos en las teorías cognitivistas que sostienen que el conocimiento lingüístico del aprendiz no se separa del modo en que lo usa, ni del modo en que está representado en su mente. Según Bialystock (1978), el cognitivismo da importancia a la percepción, la memoria, el procesamiento de la información y las estrategias de aprendizaje, a su vez influenciadas por factores externos, tanto sociales como afectivos. Por lo tanto, incluye tanto el conocimiento lingüístico como el cultural.

Siguiendo esta línea, diversos estudios han resaltado la importancia del conocimiento previo que tiene el aprendiz de conceptos y términos en su lengua materna para adoptar los comportamientos lingüísticos y culturales adecuados en la lengua extranjera. Hammerly (1991) atribuye un papel esencial a la repercusión del conocimiento previo en todo proceso de aprendizaje y, en particular, al conocimiento cultural que está limitado por la cultura origen del aprendiz. Refuerza así nuestro convencimiento de que el contexto situacional, que varía según el origen cultural del aprendiz, puede dar lugar a confusiones e interpretaciones y, consecuentemente, llevar a transferencias no solo lingüísticas sino culturales en la L2. El aprendiz sumergido en su cultura desde su nacimiento, transfiere de forma inconsciente costumbres, conceptos y comportamientos a la cultura extranjera que pueden dar lugar a malentendidos, más allá de lo estrictamente lingüístico.

Por tanto, cuando hablamos de interrelación de la lengua y la cultura, vemos que la transferencia lingüística va acompañada por la transferencia cultural y, a menudo, es inducida por ella. En este sentido, entendemos bajo transferencia lingüística / cultural el trasvase de comportamientos lingüísticos y culturales condicionados por la distancia existente entre las lenguas y culturas en contacto. 
Hemos optado por usar el término "transferencia" para evitar el de "interferencia" y su connotación negativa, puesto que en varias ocasiones, los conocimientos previos del aprendiz son precisamente los que favorecen el nuevo aprendizaje; es lo que se conoce como "transferencia positiva". A pesar de la importancia de ser conscientes de las "transferencias negativas" que pueden ocasionar un error más o menos grave en la comunicación deseada, estamos convencidos de que la gravedad del error siempre se debe analizar desde una perspectiva comunicativa y desde el parámetro de aceptabilidad, o sea, el error será más o menos grave en la medida en que afecte al mensaje y cause problemas de comunicación.

Por último, superar las distancias lingüísticas y culturales forma parte de la cotidianeidad del contacto intercultural al que se enfrenta todo aprendiz de una L2. Para ello, es necesario ser consciente no solo de los elementos lingüísticos, sino de los elementos culturales que subyacen en la comunicación, tal como hemos señalado. Esta consciencia forma parte de un conjunto de competencias y capacidades que las personas podemos desarrollar para poder hacer factible el diálogo intercultural que, al final, es el objetivo final de todo aprendizaje de lengua extranjera.

\section{Estudio empírico}

Por todo lo visto anteriormente, no solo el origen lingüístico del aprendiz, sino también el trasfondo y conocimiento cultural repercuten de forma decisiva en el comportamiento a la hora de adquirir una L2. Un ámbito en el que se toma en cuenta este precepto es el de la traducción. La distancia lingüística y cultural entre lenguas es un aspecto que incide de manera fundamental en el diseño no solo de los proyectos docentes de las asignaturas de traducción e interpretación, sino también de los de las lenguas extranjeras orientadas a la traducción y la interpretación.

El presente estudio empírico se enfoca en la influencia interlingüística e intercultural de la lengua española en la adquisición del inglés como lengua extranjera. El objetivo central es determinar si el español influye favorablemente en la adquisición de la lengua inglesa. Los sujetos de este estudio son hispanófonos, estudiantes de la asignatura Lengua BI Inglés (asignatura de $1^{\circ}$ curso) en el Grado de Traducción e Interpretación Inglés-Francés y en el Grado en Traducción e Interpretación Inglés-Alemán de la Universidad de Las Palmas de Gran Canaria, durante el curso 2014-2015. También cabe puntualizar que, paralelamente, se introduce a los alumnos al concepto de distancia lingüística y cultural en sus clases de Lingüística aplicada a la traducción, Lengua española y Documentación.

Según se señala en la programación de Lengua Inglesa en la Facultad de Traducción e Interpretación, la asignatura Lengua BI Inglés es una asignatura básica del primer semestre del primer curso del Grado en Traducción e Interpretación que consiste en "el estudio, aplicado a la traducción y a la interpretación, de la lengua inglesa en un nivel medio (B1) en sus aspectos teóricos y prácticos". Al presentar las características del nivel de competencia B1, según se define en el Marco Común Europeo de Referencia para las Lenguas, la asignatura Lengua BI Inglés tiene como objetivo principal capacitar al alumno para utilizar el idioma con cierta seguridad y flexibilidad, receptiva y productivamente, tanto en forma hablada como escrita, así como para mediar entre hablantes de distintas lenguas, en situaciones cotidianas y 
menos corrientes que requieran comprender y producir textos en una variedad de lengua estándar, con estructuras habituales y un repertorio léxico común en lengua estándar, que podrá incluir expresiones idiomáticas de uso habitual y generalizado, y que versen sobre temas generales, cotidianos o en los que se tiene un interés personal. La estructuración del programa de la asignatura de Lengua BI Inglés se considera de particular relevancia para los alumnos que acaban de dejar la educación secundaria con un nivel de conocimiento supuestamente correspondiente a un nivel A2 elevado del MER.

El instrumento utilizado para el presente estudio fue una prueba de expresión escrita en la que los alumnos tuvieron que escribir aproximadamente 150 palabras sobre el siguiente tema: The hidden forces of nature. El análisis se centra en los datos correspondientes a un grupo de 30 alumnos de la titulación de Grado de Traducción e Interpretación Inglés-Francés y otro de 30 alumnos del Grado en Traducción e Interpretación Inglés-Alemán, lo cual nos permitirá en estudios posteriores analizar la influencia de la L3 en el proceso de enseñanza / aprendizaje de la L2.

Desde etapas tempranas de la adquisición de una lengua extranjera y de manera más o menos consciente, el aprendiz diferencia los sonidos, palabras y expresiones que escucha o emplea, y 'entiende' que son de alguna manera diferentes a los correspondientes en su lengua materna. De igual forma, paulatinamente puede llegar a entender que los hablantes de la lengua que aprende perciben el mundo y estructuran su experiencia de una manera distinta a cómo lo hacen los hablantes de su lengua materna. Cierto es que algunas lenguas, normalmente cercanas geográfica, cultural, social o religiosamente, conceptualizan parte de su experiencia de manera similar aunque, como pretendemos demostrar en este trabajo, esta mayor o menor percepción de distancia entre la lengua materna y la lengua meta puede verse modificada por factores externos de diversa índole.

En su reciente trabajo acerca de la traducibilidad de las metáforas, Al-Hassnawi (2007) apunta que aquellas reflejan las experiencias cognitivas y culturales que la lengua codifica para preservar las experiencias humanas. En las metáforas, las palabras se invisten de características figurativas que están más allá de las referenciales o literales propias y por tanto el significado de sus constituyentes léxicos no puede predecirse de su significado referencial. No obstante, estas consideraciones relativas a la metáfora pueden extrapolarse al campo léxico-semántico y, en particular, a todo lo que está más allá de lo prototípico, lo no marcado o consustancial, porque, de forma similar a lo que sucede con la metáfora, todo ello puede resultar igualmente modelado y constreñido por la cultura, historia, experiencias, etc., que la lengua soporta, los significados que abarca y las asociaciones semánticas que emplea. Los mapas semánticos de las palabras de una L2 pueden incluir significados literales de los que la L1 carece, otras palabras pueden igualmente portar algún componente cultural desconocido para la L2 o puede asociarse a otras palabras que la L2 no permitiría.

Digamos que lo prototípico, es decir, lo que comparten todas las lenguas, debido a la experiencia o historia biológica común (Chitoran, 1973), quedaría fuera de estas afirmaciones y constituiría la frontera más cercana entre lenguas a efectos de distancia. En esta frontera más o menos común en la que dos lenguas comparten patrones de pensamiento, significado y actuación, podemos afirmar que es posible transferir de una a la otra o hacer traducciones equivalentes con escasa o nula pérdida de contenido o, al menos, conservando de alguna manera el mensaje de la L1. De hecho, la 
gran mayoría de las producciones escritas por los alumnos de la asignatura Lengua BI Inglés del Grado en Traducción e Interpretación de la Universidad de Las Palmas de Gran Canaria que se ha empleado en esta investigación, son fruto de la traducción directa del esquema lingüístico / cultural español a otro, supuestamente inglés, si bien de este último solamente se atiende a las formas lingüísticas. Estos intentos de interpretar literalmente de una lengua a la otra a través de la simple transferencia lingüística de significados, en muchos casos, aseguran una comunicación inteligible $\mathrm{y}$, en ocasiones, bastante exacta, porque se trata de esquemas, nociones y significados universalmente compartidas o lo que podemos llamar universales lingüísticos, sin perjuicio de que también se puedan incluir algunos universales culturales. Este último nivel incluye aquellas expresiones que transportan ideas, actitudes y valores que todos los humanos compartimos, ya que pertenecen a nuestra experiencia básica.

Veamos el siguiente ejemplo: [[AlumnoIA1-7]] "In my opinion one natural calamity is something irreparable because it destroys everything and everybody that it is in his way" En este fragmento de producción escrita, el aprendiz ha intentado transferir su idea de la L1 español a la L2 inglés de manera lineal y literal e, independientemente de sus errores gramaticales, transmite el mensaje de manera más o menos clara e inteligible. Exactamente lo mismo sucede con este otro fragmento [[AlumnoIF1-17]] "It was a blue day. My parents went to the cinema. My sister and I were in the house. We were playing with videogames." Las ideas que produce el alumno, así como las palabras que emplea pertenecen a la experiencia humana común y de ahí que su transposición a la L2 resulta fluida y correcta, errores gramaticales aparte.

No obstante, existen también traducciones que se han realizado palabra por palabra $\mathrm{y}$, como apuntamos más arriba, estas uniones llevadas a la lengua extranjera pueden producir distorsión cuando no incomprensión. El siguiente ejemplo da buena prueba de ello, [[AlumnoIA1-16]] "I was working when began to do many air and ...". El aprendiz partiendo de palabras y expresiones en L1 trata de producir un texto que en L2 resulta ininteligible, más aún si tenemos en cuenta la gramática. Para un nativo inglés, este texto carece de sentido.

Surgen problemas cuando la transferencia se lleva más allá de esquemas comunes y se intenta transferir expresiones producto de formas de pensar y de expresarse propias o específicas de otra cultura. Al igual que sucede con tales expresiones, figuras de habla o de pensamiento, también pueden surgir problemas cuando se trata de una sola palabra o de una expresión, pues por muy simple que sean, pueden incluir una carga cultural y semántica característica y específica de una cultura en particular. Tal vez sea en el capítulo de expresiones y frases hechas donde encontramos un número mayor y más llamativo de estos casos, como por ejemplo: [[AlumnoIA1-28]] “... of all life...", [[Alumno: GC107]] "Many people is in the ruin", [[AlumnoIF1-10]] “... from one moment to the other...", [[AlumnoIA1-15]] "The hidden forces of nature arrive for surprise", [[AlumnoIF1-13]] "The tree, the forest was in the floor". Pero también en el segundo caso, el que se relaciona con la carga semántica de una palabra, encontramos un gran número de desviaciones; así, por ejemplo, encontramos: [[AlumnoIF 1-12]] "More of five hundred persons helped the firemen in order to switch off the fire", [[AlumnoIA1-2]] "The forest fire is an ecology loss that also affects to the animals that live" o "external factors can do that begin a forest fire", [[AlumnoIF1-20]] "Bombs and pan cakes are flying over all the islands and...".

Según Ellis (1994), la condición de marcada o no marcada de una expresión o una palabra es uno de los condicionantes de la transferencia. Por tanto, las expresiones 
idiomáticas, alusiones clásicas o literarias, figuras y palabras que transportan elementos culturales o de conocimiento del mundo propios de una cultura en particular se consideran marcadas y, entonces, de traducibilidad difícil o indirecta, ya que precisan de la comparación de las dos lenguas y culturas. No obstante, hemos podido observar que algunos alumnos parecen no ser conscientes de esta realidad y traducen tales expresiones y palabras españolas al inglés sin percatarse de que más que un sentido literal tienen un sentido figurativo, o, en otros casos el campo semántico de la palabra abarca una extensión diferente, a veces más amplia, otras más limitada pero siempre diferente: [[AlumnoIF1-19]] " ... and the trees wear the house with wonderful colours", [[AlumnoIA1-30]] "The tornado destroyed the city in five seconds, in a one speak shape..."

En otro orden de cosa, llama la atención la percepción que los alumnos tienen en referencia a adjetivos y expresiones para describir el tiempo. Así, encontramos: [[Alumnos IF1-15]] "In Las Palmas de Gran Canaria, because of climate change, it is now freezing cold in winter. You need to put long sleeves on." Teniendo en cuenta que las temperaturas rara vez bajan de $\operatorname{los} 15^{\circ} \mathrm{C}$ en la capital de la isla de Gran Canaria, resulta obvio que las opiniones sobre el estado de bienestar personal y del tiempo están directamente influidas por la orientación cultural de los sujetos. Por este motivo resulta imprescindible tener en cuenta que las diferencias lingüísticas y culturales influyen en la manera en que los individuos perciben y comunican sus impresiones, adoptándose a los valores, criterios y puntos de vista específicos de su cultura. Los aprendices suelen creer que existe una relación simétrica entre el lenguaje y la realidad, es decir, que la realidad es la misma en todas partes y que luego son las distintas lenguas las que le ponen distintas etiquetas. Sin embargo, las relaciones entre las lenguas y las culturas son asimétricas, puesto que la realidad no siempre se percibe de la misma manera. La transferencia interlingüística está sujeta a una serie de asimetrías responsables de que un mensaje original y el mensaje meta sean necesariamente distintos. De este modo, la sociedad a la que pertenecemos condiciona la forma en que conceptualizamos la realidad, por lo que cada lengua tiene su concepción particular de la realidad y cada pueblo ve el mundo a su manera.

Otro de los condicionantes de la transferencia es lo que Kellerman (1983) denominó prototypicality y que aquí llamaremos prototipicidad. La mayor parte de los estudios de Kellerman para probar la hipótesis de la prototipicidad se desarrolló en el campo léxico-semántico. Los datos empíricos que obtuvo demuestran, por ejemplo, que los idioms son ítems que no se suelen transferir y que existe una preferencia sistemática por los usos que él denomina transparentes. Esta noción de transparencia presupone relaciones universales entre forma y significado. Las expresiones transparentes son más transferibles porque están muy cerca de lo que Odlin $(1989,142)$ llama 'core meaning' o lo que lo mismo, significado nuclear, central o consustancial a la lengua. Ese concepto de consustancialidad está obviamente relacionado con el concepto de marcado o no marcado. Kellerman (1983) afirma que los hablantes poseen percepciones sobre la estructura de su propia lengua y, en consecuencia, aporta el concepto de psycholinguistic markedness o 'marcado desde una perspectiva psicolingüística'. Posteriormente el mismo autor (1986) acuña el término prototipicidad para referirse a esa idea central. Según el mismo autor, las mencionadas percepciones que tienen los aprendices les indican lo que es transferible de su L1, esto es, lo prototípico o semánticamente transparente, y lo que no lo es y por tanto resulta intransferible. 
No obstante, estas percepciones parecen no ser iguales en todos los aprendices y encontramos algunas expresiones o palabras traducidas que llevan a pensar en la existencia de algún factor que desvirtúa esa capacidad de aprehensión de lo que es o no prototípico. Al aprender el léxico de una lengua extranjera, las diferencias culturales y conceptuales repercuten en el uso de la lengua, dando lugar a transferencias culturales y por tanto malentendidos.

\section{Aplicación didáctica}

A la hora de diseñar una programación didáctica enfocada al establecimiento de un grado de diferencia lingüística y cultural real entre la lengua inglesa y la lengua española, sugerimos dos unidades didácticas con el fin de ilustrar cómo afrontar el tratamiento de estos elementos relacionados con la distancia lingüística y cultural en la clase de lengua inglesa para alumnos de un Grado en Traducción e Interpretación. Estas unidades pretenden favorecer procesos de enseñanza-aprendizaje de la lengua inglesa en consonancia con los principios expuestos anteriormente.

La asignatura en cuestión es la de Lengua BI Inglés, asignatura básica de 6 ECTS del primer cuatrimestre del primer curso cuyo objetivo general, como lo explicamos antes, es la capacitación para utilizar el idioma con cierta seguridad y flexibilidad, en un nivel de competencia B1, según se define en el MER.

Los objetivos específicos que nos marcamos se pueden desglosar de la siguiente manera. Solo hemos detallado los que están directamente relacionados con los efectos de la distancia entre lenguas/culturas y la transferencia lingüística / cultural en el proceso de adquisición del inglés como lengua extranjera. Asimismo, hemos subrayado los aspectos que consideramos clave al respecto.

A. Desarrollar la competencia lingüística y las destrezas comunicativas, y hacer del futuro traductor e intérprete un experto en lengua inglesa.

1. Desarrollar la reflexión sobre el sistema de la lengua inglesa en sus distintos aspectos o niveles (morfosintácticos, léxicos y fonológicos) y su trascendencia comunicativa en ámbitos generales, semi-especializados y especializados.

2. A partir de la reflexión sobre el sistema de la lengua inglesa y su contraste con la lengua materna, los alumnos mejorarán sus competencias en las distintas destrezas comunicativas en dicha lengua.

3. Desarrollar la competencia discursiva o textual.

4. Desarrollar la competencia productora textual logrando propósitos comunicativos adecuados.

5. Desarrollar la competencia analítica textual (análisis del texto adecuado a la traducción).

B. Desarrollar la competencia sociocultural y hacer del futuro traductor e intérprete un experto en cultura.

1. Entender que la lengua es una herramienta de comunicación e interacción $\underline{\text { social, }}$ cuyas estructuras lingüísticas son vehículo para la transmisión de la cultura.

2. Desarrollar el conocimiento de las convenciones de la lengua, de los registros 
adecuados, de los dialectos y de la capacidad de interpretar referencias culturales.

3. Analizar las connotaciones y la carga cultural de las entidades semióticas.

4. Analizar las connotaciones y la carga cultural de determinadas unidades léxicas.

5. Analizar la carga cultural de aspectos morfosintácticos de la lengua.

6. Analizar los rasgos característicos de los géneros textuales.

C. Desarrollar la competencia contrastiva que deberá ejercer el futuro traductor e intérprete a la hora de ejercer sus tareas de mediación entre lenguas y culturas.

1. Ser conscientes de la naturaleza de las lenguas naturales como sistemas independientes con estructuras autónomas propias y características susceptibles de análisis y de comparación entre ellas.

2. Aprender a contemplar el sistema de la lengua inglesa como un término de comparación con el sistema de la lengua española, como un punto de partida de cara a descubrir dificultades y distinciones que deberán tenerse en cuenta posteriormente en las tareas de traducción e interpretación.

3. Desarrollar la competencia textual contrastiva, o sea, la habilidad de analizar las especificidades culturales de las convenciones textuales y de otro tipo de convenciones comunicativas en las dos lenguas y culturas.

4. Analizar las convenciones de la escritura: el uso de mayúsculas y minúsculas, los signos de puntuación, el tratamiento de las siglas y abreviaturas, etc.

5. Analizar dificultades de orden léxico: las palabras compuestas, los falsos amigos, etc.

6. Analizar dificultades de orden morfosintáctico: los verbos modales, el uso de las preposiciones, el uso de los tiempos verbales, el uso de adjetivos, etc.

7. Analizar dificultades de orden textual: los conectores, los marcadores característicos de los diferentes tipos textuales, la cohesión y la coherencia textual, $\underline{\text { etc }}$

D. Desarrollar las competencias estratégicas para comunicarse y aprender de manera eficaz y personalizada.

E. Desarrollar las competencias de autoaprendizaje con el fin de formar para la adaptación y el cambio.

A continuación se presenta en la siguiente tabla una unidad didáctica (unidad temática 12) enmarcada en el "Bloque 4. The world we live in" que ejemplifica el planteamiento pedagógico aquí defendido. Cada unidad didáctica, a su vez, se divide en dos grandes apartados: una enumeración de los objetivos específicos, que tienen sus raíces en los objetivos generales y específicos del conjunto de asignaturas de Lengua B Inglés para traductores que expusimos antes, y una especificación del contenido lo suficientemente precisa como para entender qué pretende transmitir cada unidad. El contenido de cada unidad temática está organizado en tres bloques principales. El primero tiene que ver con los contenidos relativos a la competencia lingüística en general, que pueden ser aspectos gramaticales, léxico-semánticos, fonéticos, fonológicos u ortográficos. El segundo bloque se ocupa de la práctica y desarrollo de las destrezas receptivas, productivas y de interacción. El tercer bloque tiene un enfoque 
cultural y profesional en el cual se consideran aspectos de la competencia sociocultural, la contrastividad, la utilización de fuentes de información, la labor del traductor, etc. Según sean los objetivos específicos de cada unidad, se hace más hincapié en un bloque de contenido o en otro.

\begin{tabular}{|c|c|}
\hline \multicolumn{2}{|r|}{ UNIT 12 - A WORLD OF DIFFERENCE } \\
\hline $\begin{array}{l}\text { CENTRAL } \\
\text { THEME }\end{array}$ & $\begin{array}{l}\text { Age and culture difference } \\
\text { Age and life stages }\end{array}$ \\
\hline $\begin{array}{l}\text { SPECIFIC } \\
\text { OBJECTIVES }\end{array}$ & $\begin{array}{l}\text { 1. To help students cope with more specialised vocabulary } \\
\text { and to encourage them to develop strategies for learning } \\
\text { vocabulary. } \\
\text { 2. To raise awareness of the importance of the context to } \\
\text { understand new vocabulary. } \\
\text { 3. To clarify confusion and revise the form of basic } \\
\text { structures used in comparisons. } \\
\text { 4. To sensitise students to set idioms of comparison. } \\
\text { 5. To sensitise students to the fact that cultural animosity is } \\
\text { often based on cultural ignorance. } \\
\text { 6. To raise awareness of the importance of understanding } \\
\text { difference and ability to communicate in cross-cultural } \\
\text { encounters. } \\
\text { 7. To help students write a convincing story about their life } \\
\text { in the future. }\end{array}$ \\
\hline $\begin{array}{l}\text { LANGUAGE } \\
\text { FOCUS }\end{array}$ & $\begin{array}{l}\text { Vocabulary content } \\
\text { Metaphors and similes } \\
\text { Idioms with like } \\
\text { Expressions using life and time } \\
\text { Grammatical content } \\
\text { Basic structures of comparison } \\
\text { The use of modifiers in comparisons } \\
\text { Other forms of comparisons (the more... the more, fewer and } \\
\text { fewer, etc.) } \\
\text { like and as as conjunctions and prepositions } \\
\text { Revision of future forms } \\
\text { Content related with phonetics and / or spelling } \\
\text { Predicting pronunciation. Melody. }\end{array}$ \\
\hline
\end{tabular}




\begin{tabular}{|c|c|}
\hline $\begin{array}{l}\text { COMMON } \\
\text { EUROPEAN } \\
\text { FRAMEWORK } \\
\text { SKILLS }\end{array}$ & $\begin{array}{l}\text { Reading } \\
\text { Text 1: You're never too old (newspaper article) } \\
\text { Reading for gist and specific information. } \\
\text { Developing one's ability to infer and speculate about a text. } \\
\text { Text 2: A series of short newspaper articles about what old } \\
\text { age means in different countries in the world. } \\
\text { Jigsaw reading. } \\
\text { Reading, writing and speaking } \\
\text { Quiz: Your pace of life } \\
\text { Students do a quiz to find out about their pace of life and } \\
\text { then discuss their answers with a partner, Students find the } \\
\text { similar lines in the quiz and compare the differences } \\
\text { Speaking and spoken interaction } \\
\text { Group discussion: students discuss the advantages and } \\
\text { disadvantages of each stage of life. } \\
\text { In pairs, students talk about people they know who are } \\
\text { 'superyoung' and discuss why these people look so young. } \\
\text { Writing and written interaction } \\
\text { Students write about what they will do when they are retired } \\
\text { in order to remain as young as possible. } \\
\text { Listening and speaking } \\
\text { Listening 1: People talking about their 'happy days' } \\
\text { Students improve their abilities to listen for specific } \\
\text { information. } \\
\text { Students discuss statements in small groups and decide } \\
\text { which stage of life the four speakers are at. } \\
\text { Listening 2: Superyoung } \\
\text { Students listen to an interview with Dr Weeks about what } \\
\text { people should do to remain young and fill in a worksheet. } \\
\text { Song: That's Life (Robbie Williams) }\end{array}$ \\
\hline $\begin{array}{l}\text { CULTURAL / } \\
\text { PROFESSIONAL } \\
\text { FOCUS }\end{array}$ & $\begin{array}{l}\text { The principle of cultural relativity. } \\
\text { To explore the issues of changing values and cultural } \\
\text { assimilation. } \\
\text { To explore the meaning of cultural symbols. } \\
\text { To identify similarities and contrasts between countries and } \\
\text { cultures. } \\
\text { Dealing with cultural difference: a challenge for translators } \\
\text { and interprets. }\end{array}$ \\
\hline
\end{tabular}

\section{Discusión y conclusiones}

En primer lugar, huelga decir que en vez de hablar de "transferencia positiva" o "transferencia negativa", preferimos hablar de una única transferencia que puede ocasionar un error más o menos grave en la comunicación deseada. La gravedad de este error siempre se debe analizar desde una perspectiva comunicativa y, por supuesto, cultural, puesto que lo que al final cuenta es el grado de aceptabilidad de lo expresado por el aprendiz, o sea, la gravedad del error se medirá según el grado en que afecte al mensaje y cause problemas de comunicación en un contexto situacional dado. 
En segundo lugar, por mucho vocabulario y reglas gramaticales que haya aprendido, un hablante no nativo de inglés puede producir oraciones perfectamente gramaticales que incluyen palabras bien escogidas pero que aún así no suenan como producciones auténticas o naturales en lengua inglesa. [[AlumnoIF1-8]] When I got out, I saw all the people out of their homes and they were very alarmed; probablemente la producción nativa sería algo como: When I left the house I saw that everyone had run out into the street and they were all very frightened.

Aún, en ciertos sectores del profesorado, así como en el concepto de muchos libros de texto, subsiste la idea de que la lengua se compone de gramática y vocabulario, y que seleccionando las palabras correctas e insertándolas en estructuras gramaticales adecuadas, podemos producir lengua como lo haría un nativo. En muchas aulas e incluso en muchos textos se presenta el vocabulario separado de la gramática y se suministran listados de palabras que los aprendices deben aprender con su traducción incluida. No digamos de esas canciones que se emplean en algunos centros en los comienzos de la enseñanza en las que se recita la palabra inglesa seguida de su correspondiente traducción en español: ventana - window, puerta - door, techo - ceiling, piso - floor. Para empezar ya le estamos enviando a los aprendices el mensaje de que la lengua inglesa es igual a la española solo que con otras palabras y que todo es traducible. Asimismo, la gramática se enseña en la pizarra o aparece en un apartado de la lección, bien al comienzo o al final de la unidad.

Por un lado, esta visión compartimentada de la lengua puede favorecer ciertas posibilidades creativas a la hora de generar producción lingüística, aunque esta no sea sino la traslación de estructuras y vocabulario españoles a una supuesta lengua inglesa. Por otro lado, la fluidez se ve absolutamente frenada cuando el proceso pasa por recurrir a las listas aprendidas y recuperar de ellas palabra tras palabra. Si el proceso natural fuera así, ¿cuánto tardaría un nativo en producir teniendo en cuenta que sus listas de palabras son muchos mayores?

En el proceso personal de descubrimiento y generación de lengua por el que, a partir del habla de sus mayores, el niño se las ingenia para reconocer unidades individuales de lengua dentro de la cadena fónica continua que oye, centraremos las diferencias que se producen entre la producción lingüística de los hablantes nativos de una lengua, en este caso el inglés, y los hablantes no nativos.

En primer lugar, dentro de la cadena fónica del discurso que se les dirige, los niños (nativos) empiezan por identificar algunas de las palabras que escucharon aisladamente, y luego emplean cadenas que han sido capaces de identificar, aunque su empleo sea incorrecto en la situación correspondiente. Igualmente, a partir de las mismas palabras aisladas $u$ otras que han sido capaces de identificar establecen las combinaciones que su inteligencia innata les permite. Por último, son capaces de diferenciar las unidades más pequeñas dentro de la cadena empleando estrategias sistemáticas ya sean propias de la lengua o del niño en particular. Este fenómeno, que se ha denominado bootstrapping, tiene diversas variedades según la técnica de solución de problemas que se emplee para ir rompiendo la cadena, en el que además se suele seguir un orden que empieza por el significado - bootstrapping semántico, el ritmo propio de la lengua - bootstrapping fonético, sus inflexiones, la gramática, - bootstrapping sintáctico.

Lo que hemos llamado bootstrapping semántico empieza por el significado básico y central de cada palabra, pero su proyección y actividad continúa sine die, porque después del escalón del significado básico se descubren otras ampliaciones 
semánticas que dependen de otras situaciones de comunicación, de otros temas, de los acompañantes que rodean a la palabra en otros contextos, del énfasis que se pone en cada situación, etc. De esta manera, los hablantes nativos adquieren un extenso corpus de ejemplos de palabras en inglés en sus propios contextos y así aprenden cómo usar cada palabra en toda su extensión y profundidad.

Por el contrario, los hablantes no nativos han recibido un input mucho más limitado, esto es, han leído o escuchado pocos ejemplos de uso de palabras en contextos naturales, incluso de las palabras más corrientes y, por consiguiente, al haber tenido menos oportunidades de aprender la manera natural en que las palabras se emplean, tienen menos posibilidades de hacer un uso correcto de ellas. Esta circunstancia puede dar a pie a posibles errores de transferencia, aunque no parece que potencie la transferencia de lo no marcado. Esta transferencia se produce por la percepción que el alumno capta a través de la metodología que sus profesores o libros de texto practican. Cuando las listas de vocabulario o la memorización descontextualizada vienen ligadas a la traducción en lengua materna, el alumno deduce que aprender lengua es aprender vocabulario y, el hecho de que no haya pasado por el proceso de identificación y descubrimiento de la lengua extranjera unido al conocimiento que, a través del aprendizaje de su lengua materna, tiene de las unidades de significado o palabras, hace que su lengua y cultura materna mediatice toda su producción.

Por tanto, el origen de la transferencia que hacen los alumnos está en la distancia entre el español y el inglés que éstos se han visto obligados a percibir. Este condicionante de distancia percibida está también presente en Ellis y es el que Kellerman denomina psicotipología. Igualmente, en sus trabajos sobre transferibilidad, Kellerman nos habla de la percepción que cada aprendiz tiene sobre la distancia que media entre la L1 y la L2. Por ejemplo, incluso antes de iniciar estudios, los hablantes españoles consideran que existen muchos más elementos transferibles al italiano que, por ejemplo, al sueco. Según Gass (1984) la transferibilidad en Kellerman es una noción relativa que depende de la distancia que entre la L1 y la L2 percibe el aprendiz, así como de la organización estructural de la L1. Esta noción de distancia percibida va cambiando a medida que el aprendiz adquiere más y más L2. Si la distancia percibida en un fenómeno psico-lingüístico, la real es puramente lingüística y cultural porque se basa en el establecimiento del grado de diferencia lingüística y cultural real entre dos lenguas. Según Selinker y Lakshmanan (1992), la congruencia estructural o al menos la similitud estructural parcial es con toda probabilidad necesaria, aunque no suficiente, para que se den la mayoría de las transferencias. Parece que cuando existen muchas semejanzas entre dos lenguas y culturas (Selinker, 2013), la transferencia lingüística y cultural resulta potenciada, ya que los aprendices tienden a aplicar reglas y conceptos de su lengua y cultura materna.

\section{Bibliografía}

Al-Hassnawi, Ali Rasheed (2007): “A Cognitive Approach to Translating Metaphors" [en línea], Translation Journal, 11(3). http://www.bokorlang.com/journal/41metaphor.htm [Consulta: 18 noviembre 2015].

Berger, Peter y Thomas Luckmann (1989): The Social Construction of Reality: A Treatise in the Sociology of Knowledge, New York / London, Penguin Books. 
Bialystock, Ellen (1978): “A theoretical model of second language learning”, Language Learning, 28(1), 69-83.

Brown, Douglas (1994): Principles of language learning and teaching, Englewood Cliffs (NJ), Prentice-Hall.

Chitoran, Dumitru (1973): Elements of English Structural Semantics, Bucureşti, EDP.

Corder, Stephen Pit (1981): Error Analysis and Interlanguage, Oxford, Oxford University Press.

Ellis, Rod, 1994: The Study of Second Language Acquisition, Oxford, Oxford University Press.

Gass, Susan (1984): “A Review of Interlanguage Syntax: Language Transfer and Language Universals”, Language Learning, 34(2), 115-132.

Hammerly, Hector (1991): Fluency and Accuracy: Toward Balance in Language Teaching and Learning, Canada, Multilingual Matters.

Hatch, Evelyn (1978): "Discourse analysis and second language acquisition", en Hatch, E. (ed.), Second language acquisition: a book of readings, Rowley, Newbury House, 401435.

Kellerman, Eric (1983): "Now you see it, now you don't”, en Gass, S. y L. Selinker (eds.), Language Transfer in Language Learning, Rowley (MA), Newbury House, 112-134

Kellerman, Eric (1986): "An eye for an eye: crosslinguistic constraints on the development of the L2 lexicon”, en Kellerman, E. y M. Sharwood Smith (eds.), Crosslinguistic Influence in Second Language Acquisition, New York, Pergamon, 35-48

Krashen, Stephen (1985): The input hypothesis: Issues and implications, London, Longman.

Long, Michael (1983): "Native speaker / non-native speaker conversation and the negotiation of comprehensible input", Applied Linguistics, 4(2), 126-141.

Odlin, Terence (1989): Language Transfer, Cambridge, Cambridge University Press.

Sapir, Edward (1954): Language. An Introduction to the Study of Speech, New York, Harvest Books.

Selinker, Larry (1972): “Interlanguage”, International Review of Applied Linguistics in Language Teaching, 10(3), 209-231.

Selinker, Larry (2013): “Interlanguage 40 Years On: three themes from here”, en Han, Z. H. y E. Tarone (eds.), Interlanguage: Forty Years Later, Amsterdam, John Benjamins, 7-26

Selinker, Larry y Usha Lakshmanan (1992): "Language Transfer and Fossilization: the Multiple Effects Principle”, en Gass, S. y L. Selinker (eds.), Language Transfer in Language Learning, Amsterdam, John Benjamins, 201-220

Soria Clivillés, Belén (1993): Teoría comunicativa de la metáfora en lengua inglesa, tesis doctoral, Universidad de Granada.

Whorf, Benjamin Lee (1956): Language, thought and reality, Cambridge (MA), MIT Press. 